\title{
VARIABILIDADE ESPACIAL DA DENSIDADE DO SOLO SOB MANEJO DA IRRIGAÇÃO
}

\author{
Spatial variability of the soil density in the irrigation management
}

\author{
Antônio Ricardo Santos de Andrade ${ }^{1}$, Ivan Amaral Guerrini², Carlos Jesús Baca Garcia ${ }^{3}$, \\ Ioeschua Katez ${ }^{4}$, Hugo Orlando Carvallo Guerra ${ }^{5}$
}

\begin{abstract}
RESUMO
Este trabalho foi desenvolvido em um solo aluvial na Estação Experimental da Embrapa Algodão, município de Souza, Estado da Paraíba, Brasil, com o objetivo de estudar a variabilidade espacial da densidade do solo. A área tem uma superfície cultivada de 17 ha e os testes de campo foram feitos em 40 pontos amostrais, locados na linha central longitudinal de cada parcela agrícola, eqüidistante de $50 \mathrm{~m}$. Na determinação da densidade do solo, foi utilizado o método do anel volumétrico, em amostras com estrutura indeformada para as profundidades de 0-30, 30-60 e 60-90 cm. A variabilidade espacial foi determinada pela técnica de estatística clássica e da geoestatística. Na análise estatística clássica, a densidade do solo para as três profundidades apresentou baixos valores de desvio-padrão e coeficiente de variação, indicando pouca variação da densidade do solo com aumento da profundidade. A densidade do solo manifestou estrutura de dependência espacial, modelada por meio de semivariogramas, os quais permitiram a interpolação por "Krigagem", apresentando estrutura esférica nas profundidades de 0-30 e 60-90 cm, com alcance de $46 \mathrm{~m}$ e estrutura exponencial com alcance de $255,10 \mathrm{~m}$ para a profundidade $30-60 \mathrm{~cm}$.
\end{abstract}

Termos para indexação: geoestatística, semivariogramas, dependência espacial, krigagem.

\begin{abstract}
This research was carried out aiming at studying soil density variability, in a soil alluvial Embrapa Algodão Experimental Station, Sousa, Paraíba, Brazil. The area has a cropped surface of 17 ha and field trials were done on 40 sampling points, placed on the central longitudinal line of each agricultural plot, distant $50 \mathrm{~m}$. On the soil density determination the volumetric ring was used, in deformed structural samples for three depths: 0-30, 30-60, and 60-90 cm. Spatial variability was determined by classical statistics and geostatistics. In the classic statistical analysis of the soil density for the three depths presented standard deviation and variation coefficient low values, indicating low variation of the soil density with the increase of the depth. The of soil density manifested structure of spatial dependence, modeled through semivariograms, it was interpolated for "Kriging", the presenting spherical structure on 0-30 and 60-90 cm depth, reaching $46 \mathrm{~m}$ for both depths exponential structure reaching $255,10 \mathrm{~m}$ for $30-60 \mathrm{~cm}$ of depth.
\end{abstract}

Index terms: geostatistics, semivariograms, spatial dependence, kriging.

(Recebido para publicação em 23 de outubro de 2003 e aprovado em 10 de março de 2005)

\section{INTRODUÇÃO}

A densidade do solo encerra uma complexidade de fatores inerente a cada local de amostragem, o que leva às dificuldades da sua avaliação. Um dos principais fatores que contribuiem para tal complexidade têm sido atribuídos à variabilidade espacial. Desde o início do século $\mathrm{XX}$, a variabilidade do solo e conseqüentemente das propriedades físico-hídricas do solo tem preocupado pesquisadores, por proporcionar diferenças no desenvolvimento, na produtividade e no manejo da irrigação, alterando resultados de pesquisas, mesmo com o solo considerado homogêneo em parcelas experimentais.

Segundo Filho et al. (2001), em razão dos atributos do solo e, em especial de a densidade não ser homogênea e apresentar dependência espacial, a variabilidade causa problemas em experimentação de campo, especialmente no manejo da irrigação. Por essa razão, a caracterização da variabilidade espacial é essencial para um entendimento melhor das inter-ralações entre atributos do solo e manejo de irrigação. Com um modelo de dependência espacial de variáveis do solo, a geoestatística possibilita a estimativa em pontos não amostrados, viabilizando o mapeamento e o zoneamento da variável. Esse procedimento pode auxiliar na melhor distribuição das parcelas e experimentos em campo, assim como o manejo mais racional da água, de fertilizantes e de defensivos agrícolas.

1. Professor Dr.Pesquisador DRC/CNPq /CCT/UEPB - Campina Grande, PB - arsa@fca.unesp.br

2. Professor Dr. Departamento de Física e Biofísica - UNESP - Campus de Rubião Junior - Botucatu, SP

3. Doutorando - UNESP/FCA - Botucatu, SP.

4. Doutor, UNESP/FCA - Botucatu, SP.

5. Professor Dr. DEAg/CCT/UFCG - Campina grande, PB. 
Entre os vários atributos do solo que interferem no manejo de irrigação e crescimento vegetal, a densidade do solo pode ser considerada a principal, sendo muito importante sua medição em projetos de irrigação e drenagem. A densidade do solo é um atributo que é afetado pela estrutura do solo, grau de compactação, manejo e tipos de culturas (Américo, 1979). A maioria das culturas é seriamente afetada quando a densidade do solo ultrapassa $1,5 \mathrm{~g} \mathrm{~cm}^{-3}$, essencialmente por duas razões: primeiro, pela falta de $\mathrm{O}_{2}$ para a respiração das raízes, devido à baixa porosidade e má drenagem e, segundo, por impedimento mecânico para o crescimento das raízes, limitando-se a zona de absorção de água e nutrientes (Souza et al, 1997).

Frazão (1981) constatou que o uso e manejo do solo têm grande influência na grandeza dos valores da densidade. Os solos superficiais sob mata e pastagens, de maneira geral, exibem baixos valores de densidade, ao passo que aqueles submetidos a cultivos contínuos mostram comumente densidades altas. $\mathrm{O}$ excessivo tráfego de máquinas agrícolas e uso repetido de implementos de cultivo causam compactação e adensamento do solo, o que, em último termo, se traduz em altos valores da densidade do solo. Também em estudos realizados por Souza et al (1997), se constatou que a movimentação de implementos agrícolas durante as diversas etapas da produção aumenta a densidade do solo e, conseqüentemente produz redução da porosidade total que, por sua vez, exercerá influência na capacidade de retenção de água, aeração, drenagem e condutividade hidráulica, afetando, assim, a produtividade das culturas.

$\mathrm{Na}$ experimentação, assim como na elaboração e manejo de projetos de irrigação, é fundamental o conhecimento da densidade do solo. Muitas vezes, pelas dificuldades no acesso aos laboratórios, custos altos e até negligência, não se realizam amostragens adequadas para uma caracterização mais detalhada da área irrigada, resultando numa escolha inadequada do sistema de irrigação, turno de rega, lâmina de água, entre outros. Dificuldades como essas favorecem a simplificação da amostragem, já que não leva em consideração a variabilidade espacial dos atributos do solo.

É por essa razão que a geoestatística se apresenta como nova técnica adicional, que leva em consideração as distribuições espaciais das amostras, permitindo definir o raio de dependência espacial entre elas. Essa dependência ou correlação espacial entre amostras pode ser verificada pelas semivariâncias (Vieira et al, 1983).
O semivariograma é a representação gráfica do ajuste de modelos matemáticos aos dados observados, de onde se definem os parâmetros necessários para a estimação de valores para locais não amostrados, por meio da técnica de "krigagem" (Souza et al, 1997). A variabilidade espacial pode ser representada por mapas de isolinhas e mapas de superfícies que são confeccionados a partir de estimativas da variável em locais não medidos pela técnica de interpolação conhecida como "Krigagem" (Takeda, 2000). As informações mostradas nesses mapas são muito úteis para melhor entender a variabilidade da densidade do solo em campo e para identificar áreas que necessitam de diferentes tipos de manejo.

Com o presente trabalho teve-se como objetivo estudar a variabilidade espacial da densidade do solo, empregando técnicas da estatística clássica e geoestatística sob área irrigada, determinando a dependência espacial por meio de semivariogramas e construindo mapas de isolinhas e superfícies com uso da técnica de interpolação da krigagem.

\section{MATERIAL E MÉTODOS}

O trabalho foi conduzido na Estação Experimental da Embrapa/Algodão, localizada no Perímetro irrigado de São Gonçalo, no município de Souza na Bacia do Alto Piranhas, no Estado da Paraíba. Os solos são do tipo Aluvial Eutrófico, fase caatinga hiperxerofila, relevo plano.

Utilizou-se uma amostragem sistemática, que consistiu em estabelecer um plano de amostragem seguindo um critério linear, com distâncias pré-fixadas entre amostras. A área foi dividida em 10 parcelas experimentais, sendo no centro de cada uma dessas traçada uma linha imaginária equiidistante a cada 50 metros. Em cada linha, os pontos de amostragem foram distribuídas com distâncias de $50 \mathrm{~m}$ a partir da linha de irrigação principal, totalizando um número de 40 pontos amostrais (Figura 1). Trincheiras de dimensões $2 \times 2 \times 2 \mathrm{~m}$ foram abertas em cada ponto amostrado, destinadas à coleta de amostras do solo com estrutura indeformada, objetivando determinar a densidade do solo. Em cada local, foi determinada a densidade pelo método do anel volumétrico de bordas de $5,10 \mathrm{~cm}$ de altura e $5,0 \mathrm{~cm}$ de diâmetro de forma a proporcionar um volume de 100 $\mathrm{m}^{3}$, sendo a extração das amostras com estrutura indeformada para as três camadas de profundidades 0-30, 30-60 e 60-90 cm. 


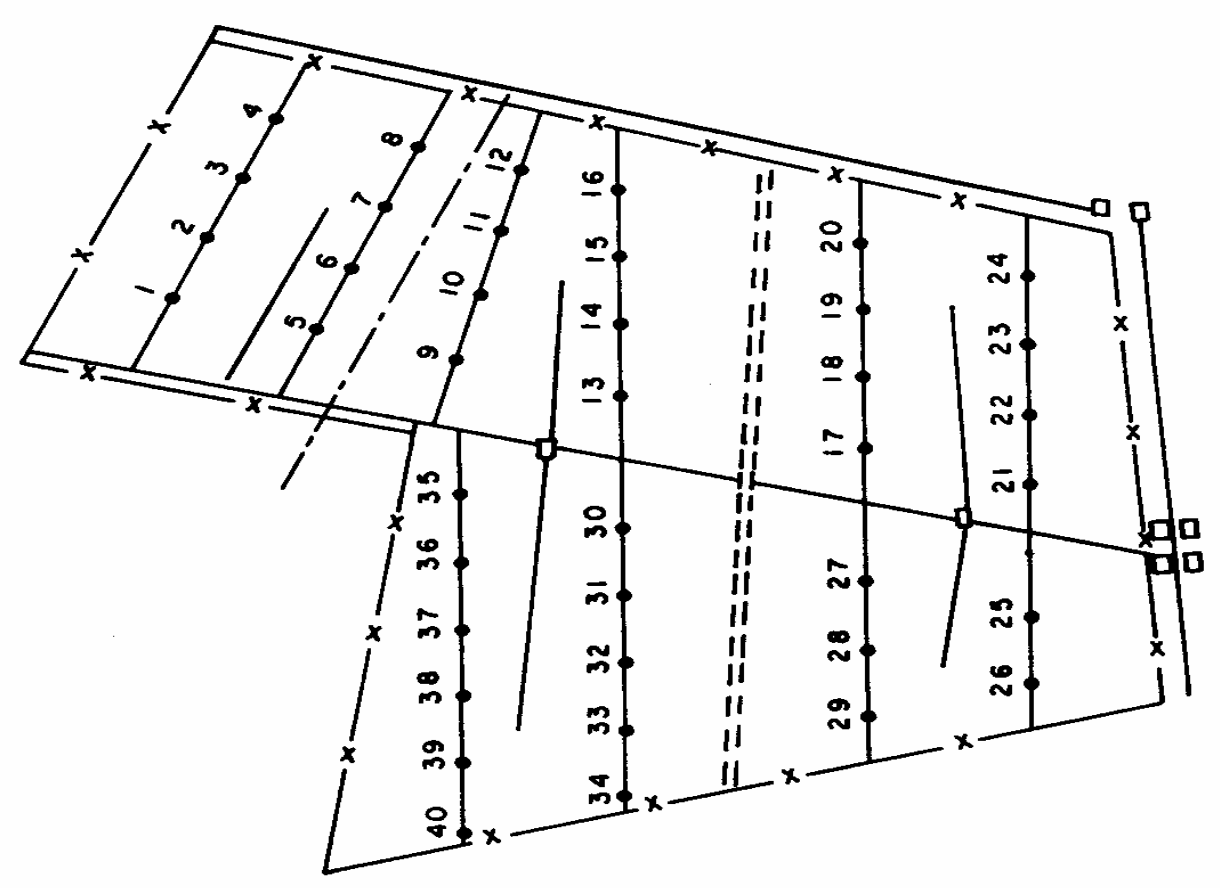

FIGURA 1 - Croqui da amostragem sistemática.

Por meio da estatística descritiva, determinaram-se a média aritmética, a mediana amostral, a variância, o desvio-padrão amostral e o coeficiente de variação. Esse procedimento foi importante para o conhecimento preliminar da distribuição da densidade do solo na área. Pela geoestatistica, analisaram-se a estrutura e a dependência espacial, que é identificada pelo estudo da forma do semivarioagrama com seus respectivos parâmetros (Ao, $\mathbf{C o}, \mathbf{C}$ e C1). A dependência espacial é quantificada pelo parâmetro alcance (Ao), o erro comedido devido à distância de amostragens, definido pelo efeito pepita $(\mathbf{C o})$ e o ponto onde toda semivariância da amostra dos dados é de influência aleatória é medida variância estrutural ou espacial $(\mathbf{C l})$ e o patamar $(\mathbf{C})$ corresponde aproximadamente ao valor da variância total da variável em estudo, é obtido de $\mathbf{C}=(\mathbf{C o}+\mathbf{C l})$. A estrutura e a dependência espacial entre as observações podem ser expressa pelo semivariograma, estimado pela seguinte expressão:

$$
\bar{\gamma}(\mathrm{h})=\frac{1}{2 \mathrm{~N}(\mathrm{~h})} \sum_{\mathrm{i}=1}^{\mathrm{N}(\mathrm{h})}[\mathrm{Z}(\mathrm{xi})-\mathrm{Z}(\mathrm{xi}+\mathrm{h})]^{2}
$$

sendo: $\bar{\gamma}(\mathrm{h})=$ valor do semivariância estimado para a distância de separação $(\mathrm{h})$ entre a medidas, $\mathrm{Z}\left(\mathrm{x}_{\mathrm{i}}\right)=$ valor da variável para posição $x_{i}$ não estimado (verdadeiro) considerada como uma variável aleatória, função da posição da amostragem $\mathrm{x}, \mathrm{Z}\left(\mathrm{x}_{\mathrm{i}}+\mathrm{h}\right)=$ valor da mesma variável na posição $\mathrm{x}_{\mathrm{i}}+\mathrm{h}$ em qualquer direção, $\mathrm{h}=$ é a distância de separação entre as medidas e $\mathrm{N}(\mathrm{h})=$ número de pares experimentais de dados medidos de $\mathrm{Z}\left(\mathrm{x}_{\mathrm{i}}\right)$ e $\mathrm{Z}\left(\mathrm{x}_{\mathrm{i}}+\mathrm{h}\right)$. O gráfico de $\bar{\gamma}(\mathrm{h})$ versus os valores correspondentes de $\mathrm{h}$, chamado semivariograma, é uma função do vetor $\mathrm{h}$, e, portanto, depende tanto de sua magnitude como da direção de $h$.

Visando a definir limites distintos de dependência espacial para os atributos do solo, Zimback (2001) adaptou os limites propostos por Cambardella et al. (1994) e propôs as seguintes classificações: i) $(\mathrm{Cl} / \mathrm{C}) .100 \leq 25 \%$ indicando variável espacial fraca dependente, ou seja, alta variabilidade espacial da variável; nesse caso, não há restrição quanto à aplicação da estatística clássica para qualquer distância ii) $25 \%$ $<(\mathrm{Cl} / \mathrm{C}) .100 \leq 75 \%$ indica variável moderadamente dependente e, iii) $(\mathrm{Cl} / \mathrm{C}) .100>75 \%$ indicando variável espacial fortemente dependente, ou seja, pequena variabilidade espacial da propriedade.

Modelos matemáticos devem ser ajustados aos semivariogramas, os quais permitem visualizar a natureza 
da variação espacial da densidade do solo, além de serem necessários para outras aplicações, como, por exemplo, krigagem.

Os seguintes modelos matemáticos mais usados podem ser ajustados aos semivariogramas:

a) Modelo esférico:

$$
\begin{aligned}
& \bar{\gamma}(\mathrm{h})=\mathrm{Co}+\mathrm{Cl}\left[\frac{3}{2}\left(\frac{\mathrm{h}}{\mathrm{Ao}}\right)-\frac{1}{2}\left(\frac{\mathrm{h}}{\mathrm{Ao}}\right)^{3}\right], 0<\mathrm{h}<\mathrm{Ao} \\
& \bar{\gamma}(\mathrm{h})=\mathrm{Co}+\mathrm{Cl}, \mathrm{h} \geq \mathrm{Ao}
\end{aligned}
$$

b) Modelo exponencial:

$\bar{\gamma}(\mathrm{h})=\mathrm{Co}+\mathrm{Cl}\left[1-\exp \left(-3 \frac{\mathrm{h}}{\mathrm{Ao}}\right)\right], 0<\mathrm{h}<\mathrm{d}$

em que d é a máxima distância na qual o semivariograma é definido;

$$
\begin{aligned}
& \text { c) Modelo gaussiano: } \\
& \bar{\gamma}(\mathrm{h})=\mathrm{Co}+\mathrm{Cl}\left[1-\exp \left(-3\left(\frac{\mathrm{h}}{\mathrm{Ao}}\right)^{2}\right)\right], 0<\mathrm{h}<\mathrm{d}
\end{aligned}
$$

A analise da dependência espacial determinada pelo semivariograma foi obtida com o auxilio do programa GS+ "Geostatistical for Environmental Sciences", Versão 5.0 (GS+ 2000), que utiliza os valores das variáveis de densidade do solo associados às suas respectivas coordenadas de campo.

Ajustar modelos matemáticos aos semivariogramas é um procedimento subjetivo. Porém, a qualidade de ajuste pode ser verificada por meio da técnica de validação cruzada. Para a densidade do solo que apresenta dependência espacial, espera-se que o semivariograma comece com um baixo valor, denominado efeito denominado alcance (Ao), que corresponde ao limite da dependência espacial e também define o raio máximo para interpolação por krigagem. A partir do alcance, a variável apresenta-se espacialmente independente e a semivariância tende a se estabilizar em torno de um valor denominado de patamar $(\mathbf{C o}+\mathbf{C l})$. A decisão pelo melhor modelo e respectivos coeficientes foi tomada com base na validação cruzada.

Existindo a dependência ou correlação espacial, as estimativas para pontos não observados são obtidas pela técnica da krigagem. Essa é uma técnica de interpolação não tendenciosa, que possui variância mínima, sendo cada estimativa obtida pelo cálculo de uma média ponderada de um conjunto de observações ao redor de uma vizinhança (Andrade, 2002), em que a estimativa
$\bar{Z}\left(x_{0}\right)$ associada à posição $x_{0}$, pode se obtida pela expressão:

$\overline{\mathrm{Z}}\left(\mathrm{x}_{\mathrm{o}}\right)=\sum_{\mathrm{i}=1}^{\mathrm{N}} \lambda_{\mathrm{i}} \mathrm{Z}\left(\mathrm{x}_{\mathrm{i}}\right)$

sendo: $\bar{Z}\left(\mathrm{x}_{\mathrm{o}}\right)=$ valores estimados da variável em estudo, $\mathrm{x}_{\mathrm{o}}=$ ponto a ser estimado, $\mathrm{N}=$ número de vizinhos utilizado na estimativa $\overline{\mathrm{Z}}\left(\mathrm{x}_{\mathrm{o}}\right), \lambda_{\mathrm{i}}=$ pesos ponderados associados a cada valor medido de $\bar{Z}\left(\mathrm{x}_{\mathrm{o}}\right)$.

A krigagem dos valores de densidade do solo, que consiste numa interpolação desses valores entre os pontos amostrados, foi efetuado pelo módulo Interpolação - Krigagem, do software GS+ (G+, 2000). Por meio da técnica de interpolação da Krigagem foram construído os mapas de isolinhas e de superfície em três dimensões (3D) representativo da distribuição espacial da densidade do solo, com o auxílio do programa Surfer versão 7.0 (Golden Software, 2001).

\section{RESULTADOS E DISCUSSÃO}

Na Tabela 1 observam-se os momentos estatísticos dos dados de densidade nos 40 pontos amostrados, nas três profundidades. Observa-se que os valores médios encontrados para as três profundidades são semelhantes, indicando ausência de variação espacial com aumento da profundidade. Porém, as medidas da estatística clássica, a variância, o desvio-padrão e o coeficiente de variação indicam pequena variabilidade dessa propriedade do solo. Os resultados estão coerentes com os obtidos por Salviano et al. (1998), que encontraram significativas variabilidades da densidade do solo nas camadas mais profundas.

Na Figura 2 podem ser verificados os semivariogramas experimentais para as profundidades do solo estudadas, de 0-30 e 60-90 cm ajustados pelo modelo esférico e profundidade $30-60 \mathrm{~cm}$ que foi ajustado pelo modelo exponencial.

Analisando os semivariogramas, observa-se dependência espacial para a densidade do solo, com efeito pepita igual 0,0004, 0,006 e 0,0018 para as profundidades 0-30, 30-60 e 60-90 cm, respectivamente. Os semivariogramas apresentaram também certa aleatoriedade dos dados, com valores do "efeito pepita" próxima do patamar, caracterizando valores altos da semivariância estimada, consequientemente, menor confiabilidade nos valores estimados. 
TABELA 1 - Momentos estatísticos dos dados da densidade, obtidos a partir de 40 medições para as três profundidades do solo.

\begin{tabular}{lc|c|c}
\hline \multirow{2}{*}{ Parâmetros estatísticos } & \multicolumn{3}{c}{ Densidade do solo $\left(\mathbf{g c m}-{ }^{\mathbf{3}}\right)$} \\
\cline { 2 - 4 } & $\mathbf{0 ~ - 3 0}$ & $\mathbf{3 0} \mathbf{- 6 0}$ & $\mathbf{6 0} \mathbf{- 9 0}$ \\
\hline Média & 1,52 & 1,52 & 1,50 \\
Variância & 0,006 & 0,015 & 0,011 \\
Desvio -padrão & 0,08 & 0,12 & 0,10 \\
Coeficiente de variação & 5,15 & 7,91 & 7,02 \\
\hline
\end{tabular}
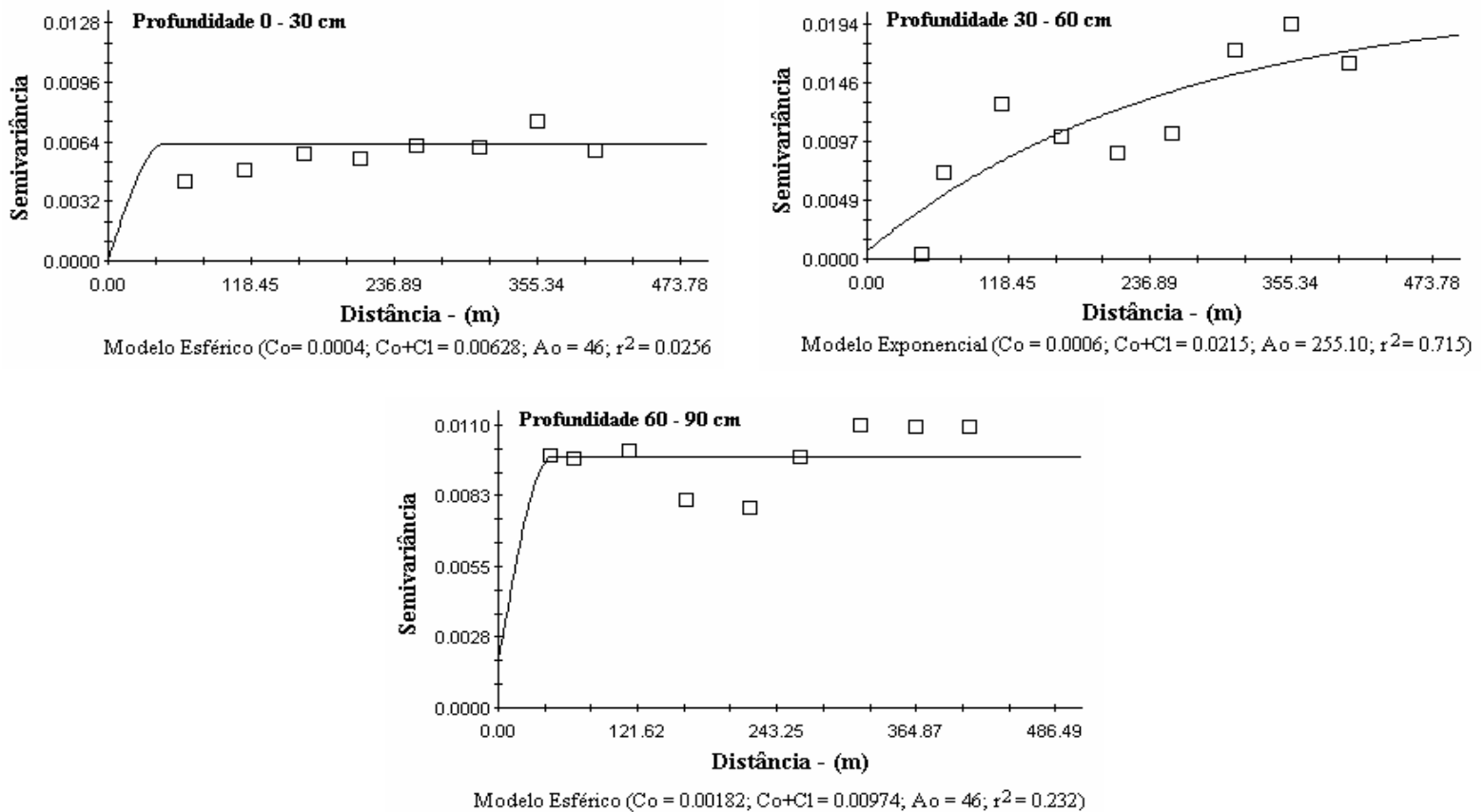

FIGURA 2 - Semivariogramas experimentais e teóricos para as três profundidades estudas.

A partir dos ajustes dos modelos aos semivariogramas experimentais, foram determinados os parâmetros para as três profundidades, necessários para a estimação de valores para locais não amostrados, pela técnica de "krigagem", apresentado na Tabela 2.

Analisando os resultados da Tabela 2, pode-se notar que os efeitos pepita para densidade do solo correspondem a 99,4; 96,9 e 81,3\% (variância estrutural/efeito pepita) da variabilidade total nas suas respectivas profundidades, concluindo-se que nessa área ocorre forte variabilidade espacial a distâncias menores que 50 metros, segundo os critérios propostos por Cambardella et al. (1994), adaptado por Zimback (2001). Os parâmetros de alcance encontrados foram iguais a $46 \mathrm{~m}$ para as profundidades de 0-30 e 60-90 cm, e $255,10 \mathrm{~m}$, para a profundidade 30-60 $\mathrm{cm}$. Segundo Andrade (2002), o alcance corresponde ao conceito da zona de influência ou dependência espacial de uma amostra, marcando a distância a partir das quais as amostras tornam-se independentes.

Portanto, o alcance da dependência espacial da densidade do solo representa a distância na quais os pontos de amostragem apresentam-se correlacionados, sendo um parâmetro de fundamental importância para planejamento e avaliações de experimentos de irrigação, não somente para determinar a intensidade de amostragem, como também ajudar no planejamento de programas de irrigação, uma vez que detecta até onde uma variável em estudo apresenta dependência espacial.

Com os modelos de semivariogramas ajustados, realizou-se a krigagem da área amostrada, permitindo tomadas de decisões e tratamentos diferenciados para regiões mapeadas. Nas Figuras 3 e 4, estão representados as isolinhas e os mapas de superfície da densidade do solo para as três profundidades. 
TABELA 2 - Os parâmetros dos semivariogramas teóricos (modelos) encontrados pela análise da geoestatística da densidade do solo.

\begin{tabular}{c|c|c|c|c|c|c}
\hline Profundidade & Co & C & Cl & $\mathbf{( C l / C ) 1 0 0}$ & Ao & Modelo \\
\hline $0-30$ & 0,0004 & 0,00628 & 0,00588 & 0,994 & 46,00 & Esférico \\
$30-60$ & 0,0006 & 0,02152 & 0,02092 & 0,969 & 255,10 & Exponencial \\
$60-90$ & 0,0018 & 0,00974 & 0,00792 & 0,813 & 46,00 & Esférico \\
\hline
\end{tabular}

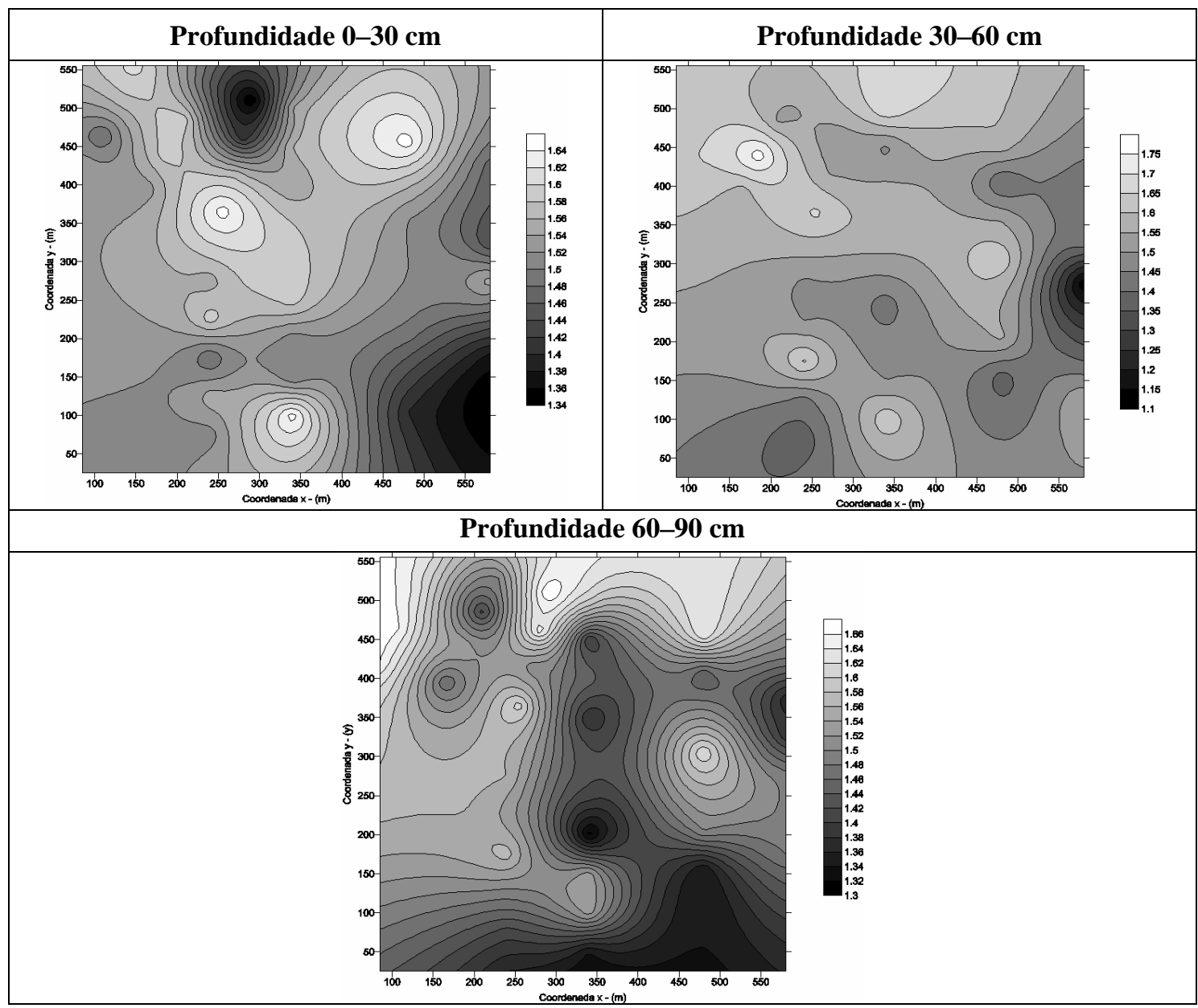

FIGURA 3 - Mapa de isolinha dos dados da densidade para as três profundidades do solo.

$\mathrm{Na}$ Figura 3 verifica-se a disposição da densidade do solo, onde as pequenas áreas com ocorrência de concentrações de linhas de contorno fechadas indicam a ocorrência de variações nos valores da densidade do solo, isto é, flutuações nos valores em intervalo de espaço amostral pequenos. Esse aspecto pode ser muito bem visualizado pela presença de picos mostrados nos mapas de superfície (Figura 4). Em grande parte da área, a densidade encontra-se entre 1,30 a 1,68 $\mathrm{gcm}^{-3}$ para as profundidades de 0-30 e
$60-90$ e de 1,25 a 1,70 para a profundidade $30-60 \mathrm{~cm}$, e uma pequena parte se encontra na faixa de 1,10 a 1,10 $\mathrm{gcm}^{-3}$.em todas as profundidades. Nota-se também uma maior densidade na profundidade $60-90 \mathrm{~cm}$, que por meio da Figura 3, corresponde à parte baixa da superfície, em direção a coordenada $\mathrm{X} \mathrm{e}$, para região superior do terreno, observam-se baixos valores na densidade do solo. Esse aspecto pode ser melhor visualizado pela presença de picos (e/ou depleções) nos mapas tridimensional (Figura 4). 


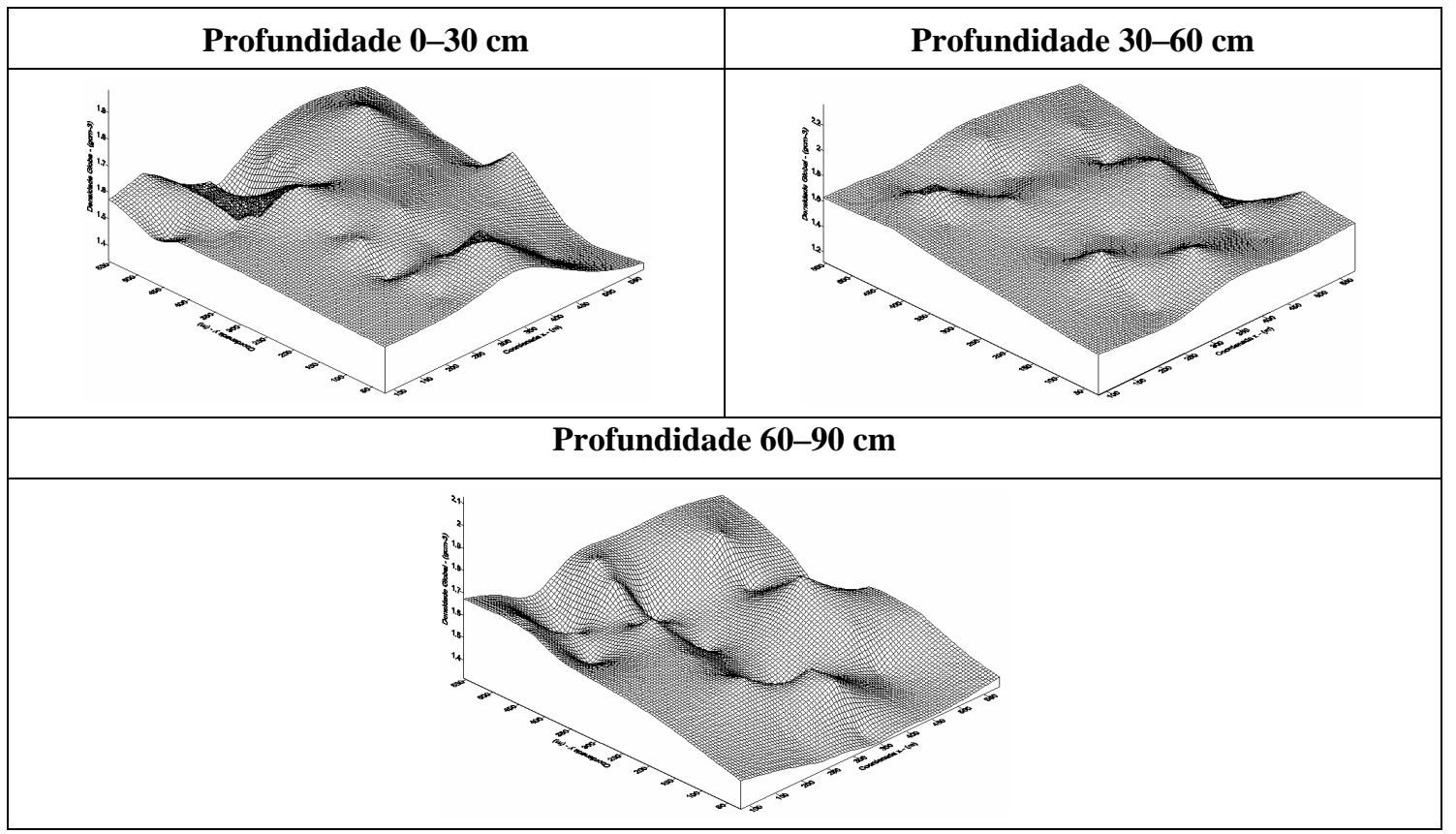

FIGURA 4 - Mapas de superfície da densidade do solo para as três profundidades.

Os mapas de superfície constituem uma ferramenta de melhor visualização da variabilidade dos valores estimados pelo processo de krigagem, representados no campo tridimensional. Na Figura 4, pode-se notar o comportamento da densidade com maiores valores concentrados na parte superior da superfície, correspondendo à área de cota mais elevada da área experimental, crescendo em direção à coordenada Y.

\section{CONCLUSÕES}

a) Os baixos valores de variância, desvio-padrão e coeficiente de variação indicam que a densidade do solo apresenta fraca variação espacial com aumento da profundidade.

b) Analisando os semivariogramas, observa-se dependência espacial para a densidade do solo para as profundidades de 0-30, 30-60 e 60-90 cm, como também uma certa aleatoriedade dos dados medidos, com valores do efeito pepita muito próximos do patamar, caracterizando valores altos da variância estimada.

c) Pela análise geoestatística, pode-se detectar a existência de estrutura ou grau de organização na distribuição espacial dos parâmetros estudados; a partir daí, pela técnica do Krigagem, é possível mapear esses parâmetros e identificar, no campo, o local que representaria os valores médios da densidade do solo.

d) A grande importância dos mapas construídos permitiu o estabelecimento de subáreas com características de densidade do solo semelhantes, nas quais se pode realizar, de maneira mais eficiente, o planejamento e manejo da irrigação.

\section{REFERÊNCIAS BIBLIOGRÁFICAS}

AMÉRICO, J.L. Características Físicas e fator de erobilidade de quatro solos representativos do município de São Mamede Paraíba-Areia. 1979. 89p. Dissertação (Mestrado em Agronomia) - Universidade Federal da Paraíba UFPB, Areia/PB, 1979.

ANDRADE, A. R. S. Aplicação da Teoria fractal e da geoestatística na estimativa da condutividade hidráulica saturada e do espaçamento entre drenos. 2002. 181p, Botucatu/SP, Tese (Doutorado em Agronomia) - Faculdade de Ciências Agronômicas, Universidade Estadual Paulista, Botucatu, 2002.

BERG, M.V.D. \& KLAMT, E. Variabilidade espacial de características de solos na região do Planalto Médio, Rs: II. Análise da semivariância e da variância. Revista Brasileira de Ciência do solo, Campinas,v.21, p.40-48, 1997.

CAMBARDELLA, C.A.; MOORMAN, T.B.; NOVAK, J.M. Field-scale variability of soil properties in central Iowa soils. Soil Science Society of American Journal, Madison, v.58, p.1501-1511, 1994. 
FILHO, M.A.C.; COELHO, R.D.; GONÇALVES, A.C.A. Variabilidade de varáveis físico-hidrícas do solo em um pomar de lima ácida Tahiti, irrigada por microaspersão . Revista Brasileira de Engenharia Agrícola e Ambiental, Campina Grande, PB, v.6, p.239-246, 2001.

FRAZÃO, A.(1981). Características físicas e químicas de um latossolo vermelho amarelo submetido a diferentes sistemas de manejo. 1981. 87p. Dissertação (Mestrado em EnGenharia Civil) - Universidade Federal da Paraíba UFPB, Campina Grande/PB. 1981.

GS+. Geoststistical for Environmental Sciences. Version 5.0. Michigan: Gamma Design Sotware, 2000.

GOLDEN SOFWARE - SURFER FOR WINDOWS. Superface mapping system. New York, verson 7, Inc., 2001.

SALVIANO, A.A.C.; VIEIRA, S.R.; SPAROVEK, G. Variabilidade espacial de atributos de solo e de crotolaria juncea L. em área severamente erodida. Revista
Brasileira de Ciência do solo, Campinas, v.22, p.115122, 1998.

SOUSA, L.S.; COGO, N.P.; VIEIRA, S.R. Variabilidade de propriedades físicas e químicas do solo em pomar cítrico. Revista Brasileira de Ciência do solo, Campinas, v.21, p.367-372, 1997.

TAKEDA, Y. E. Variabilidade espacial de atributos físicos do solo. 2000. 87p. Tese (Doutorado em Agronomia) - Faculdade de Ciências Agronômicas, Universidade Estadual Paulista, Ilha Solteira, 2000.

VIEIRA， S.R.; HATFIELD， L.; NIELSEN, D.R.; BIGGAR, J.W. Geostatical Theory and Application to variability of some agronomical properties. Hilgardia, v.51, n.3, p.116-123, 1983.

ZIMBACK, C.R.L. Análise especial de atributos químicos de solos para fins de mapeamento da fertilidade do solo. 2001.114p. Tese (livre-Docência) - Faculdade de Ciências Agronômicas, Universidade Estadual Paulista, Botucatu, 2001. 